\title{
eOftalmología: estado actual y tendencias futuras
}

\section{eOphthalmology: current state and future tendencies}

\author{
J. Andonegui ${ }^{1}$, L. Serrano ${ }^{2}$, A. Eguzkiza ${ }^{2}$
}

\section{RESUMEN}

La eSalud se puede definir como el uso de las tecnologías de la información y las comunicaciones para proporcionar o apoyar un diverso grupo de actividades relacionadas con la atención en salud. Si este concepto se traslada a la atención oftalmológica sería lo que denominamos como eOftalmología. En este artículo se describe el estado actual de los modelos de eOftalmología en el cribado de la retinopatía diabética y el diagnóstico y el seguimiento del glaucoma crónico y la degeneración macular asociada a la edad. También se definen los requerimientos tecnológicos necesarios para implantar este tipo de modelos de asistencia, se discuten las ventajas derivadas de los mismos y se hace una previsión del impacto que la eOftalmología puede tener en el futuro de la asistencia sanitaria.

Palabras clave. eOftalmología. Glaucoma. Diabetes. Degeneración macular.

\begin{abstract}
eOphthalmology can be defined as the use of information and telecommunications technologies to provide or support a group of activities related to ophthalmic care. The same concept applied to ophthalmic care would be eOphthalmology. This paper describes the current state of eOphthalmology-based models in diabetic retinopathy screening and in the diagnosis and follow-up of chronic glaucoma and age-related macular degeneration. Furthermore, the main advantages of these models and the technological requirements needed for their implementation are described. Finally, a prediction of the impact of eOphthalmology on the future of health care is offered.
\end{abstract}

Key words. eOphthalmology. Glaucoma. Diabetes. Macular degeneration.
1. Servicio de Oftalmología. Hospital de Navarra. Pamplona.

2. Departamento de Ingeniería Eléctrica y Electrónica. Universidad Pública de Navarra. Pamplona.

Recepción: 21 de octubre de 2009

Aceptación provisional: 19 de enero de 2010

Aceptación definitiva: 4 de febrero de 2010

\section{Correspondencia}

José Andonegui Navarro

Servicio de Oftalmología. Hospital de Navarra

Irunlarrea, 3

31008 Pamplona

Tfno. 848422194

E-mail: jandonen@cfnavarra.es 


\section{INTRODUCCIÓN}

La eSalud se puede definir como el uso de las tecnologías de la información y las comunicaciones para proporcionar o apoyar un diverso grupo de actividades relacionadas con la atención en salud. El mismo concepto pero aplicado a la atención oftalmológica sería la eOftalmología ${ }^{1}$. La eOftalmología puede abarcar diversos aspectos de la práctica médica como son establecer diagnósticos, realizar tratamientos, actividades preventivas, educacionales o de investigación. La eOftalmología hace la práctica médica independiente del tiempo y del lugar, permite a los especialistas una mejor organización de su tiempo y hace la asistencia médica más accesible para los pacientes.

La puesta en marcha de los sistemas de eOftalmología requiere equipos periféricos para la captación de datos e imágenes, sistemas centralizados de almacenamiento, gestión y transmisión de archivos digitales, sistemas informatizados de historia clínica para manejar los resultados y protocolos para organizar la asistencia ${ }^{2}$. La eOftalmología puede emplearse para el cribado de la retinopatía diabética, el examen del segmento anterior del ojo, el cribado del glaucoma, la realización de consultas de baja visión o incluso para proporcionar ayuda a distancia durante intervenciones quirúrgicas. Estos sistemas pueden ser empleados en tiempo real, por ejemplo para la asistencia quirúrgica o en diferido, como se hace en el cribado de la retinopatía diabética. En tiempo real son más costosos, requieren medios tecnológicos más complejos y mayor ancho de banda para la transmisión.

A lo largo de este artículo se describe el estado actual de los sistemas de eOftalmología en la retinopatía diabética, el glaucoma crónico y la degeneración macular asociada a la edad (DMAE), se repasan los requerimientos tecnológicos necesarios para la implantación de estos sistemas y se discuten las ventajas asociadas a esta forma de asistencia. Para finalizar, se intenta predecir cómo puede ser el futuro de la asistencia sanitaria con la implantación de modelos de eSalud.

\section{ESTADO ACTUAL}

\section{Retinopatía diabética}

La retinopatía diabética es una de las complicaciones más temidas de la diabetes mellitus y la principal causa de pérdida visual en las personas en edad laboral de los países de nuestro entorno. Para prevenir y tratar de forma temprana las alteraciones provocadas en la retina por la diabetes, se recomienda una revisión anual del fondo de ojo $^{3}$. No obstante, la elevada prevalencia de esta enfermedad hace que, en la práctica, un elevado porcentaje de estos pacientes no puedan acudir a ser revisados dentro de los plazos indicados. Para paliar este problema, se ha propuesto la utilización de los sistemas de retinografía no midriática para la exploración del fondo de ojo ${ }^{4}$. Estos sistemas consisten en la realización y almacenamiento, por parte de enfermeras o de otros trabajadores sanitarios, de fotografías digitales de la retina de los pacientes diabéticos que posteriormente son examinadas por oftalmólogos o por médicos de atención primaria $^{5}$ para decidir quiénes de ellos se encuentran afectados (Figs. 1 y 2).

La fiabilidad de la retinografía no midriática para detectar las alteraciones provocadas por la retinopatía diabética es muy similar a la de los métodos clásicos de exploración retiniana ${ }^{6,7}$. Además presenta varias ventajas, entre ellas, que resulta más económica, emplea menos tiempo por paciente, puede ser aplicada a poblaciones físicamente alejadas de los centros de atención especializada y no precisa dilatación pupilar. Por otra parte, diferentes autores han valorado la concordancia existente entre la interpretación de las imágenes digitales de la retina y la exploración directa de la misma en las consultas de oftalmología, encontrando que en general la correlación entre ambos sistemas es muy alta, lo que avalaría la utilidad del sistema de retinografía no midriática como método de cribado y detección de la retinopatía diabética ${ }^{8-10}$. La mayor parte de los estudios que han analizado esta técnica han encontrado niveles de sensibilidad superiores al $80 \%$ y niveles de especificidad mayores del $90 \%$, lo cual 
respalda su idoneidad como método de cribado $^{11,12}$. Además, existen trabajos que han demostrado que la retinografía no midriática, comparada con los métodos clásicos de exploración, es superior en relación coste-efectividad para la detección de la retinopatía diabética. Esto ocurre tanto si la medición se hace en años de vida ajustados por calidad (AVAC) ${ }^{12}$ como si se compara de forma directa el coste por paciente ${ }^{11,13}$.

Figura 1. Arriba: retinógrafo no midriático. Consta de una cámara de fotos digital (izquierda) conectada a un ordenador (derecha) que procesa las imágenes y posteriormente las envía a un servidor externo. Abajo: una enfermera realiza las retinografías a una paciente diabética.

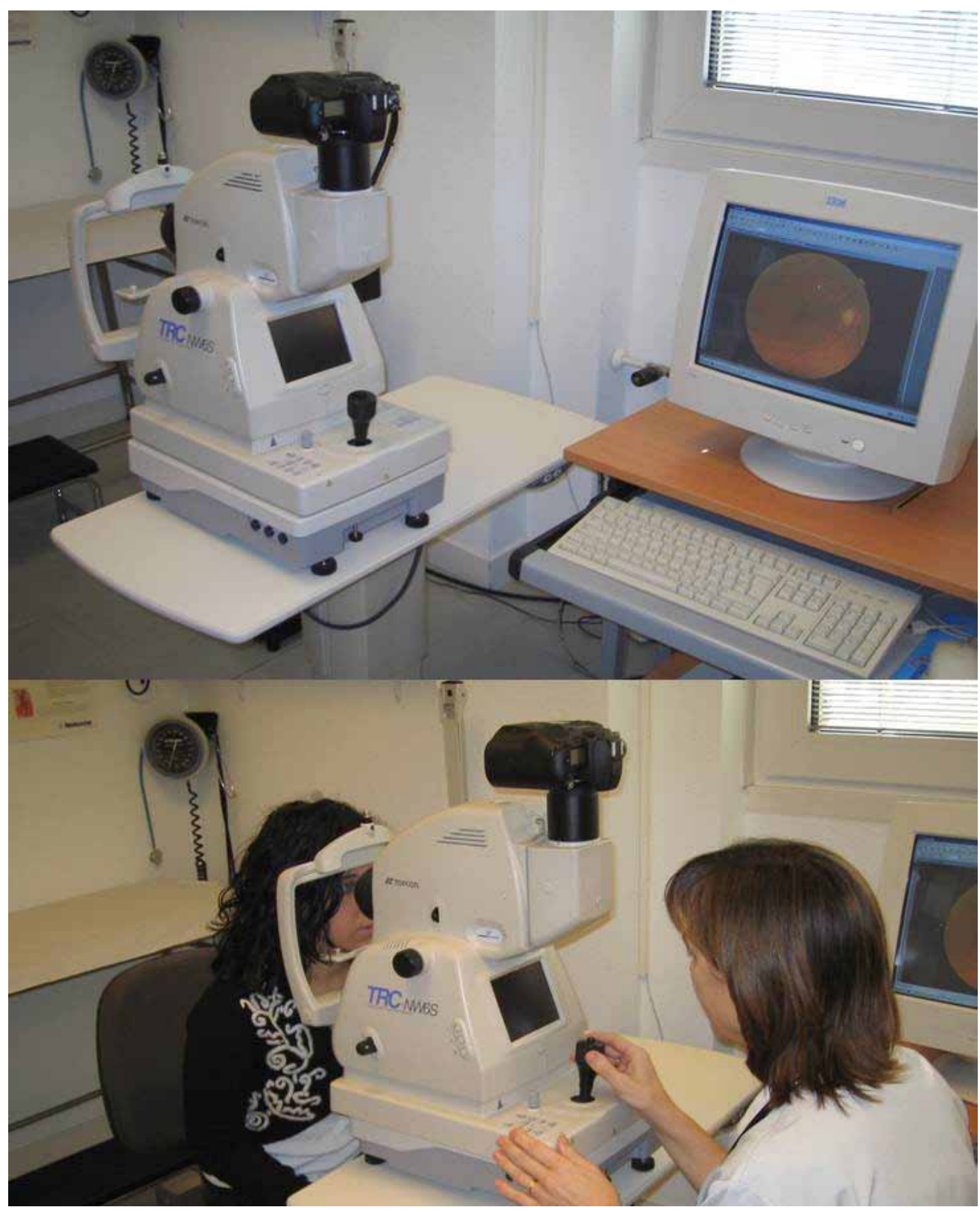


Figura 2. Retinografías de pacientes diabéticos. Arriba: retinografía normal. Abajo: retinografía con abundantes signos de retinopatía diabética.

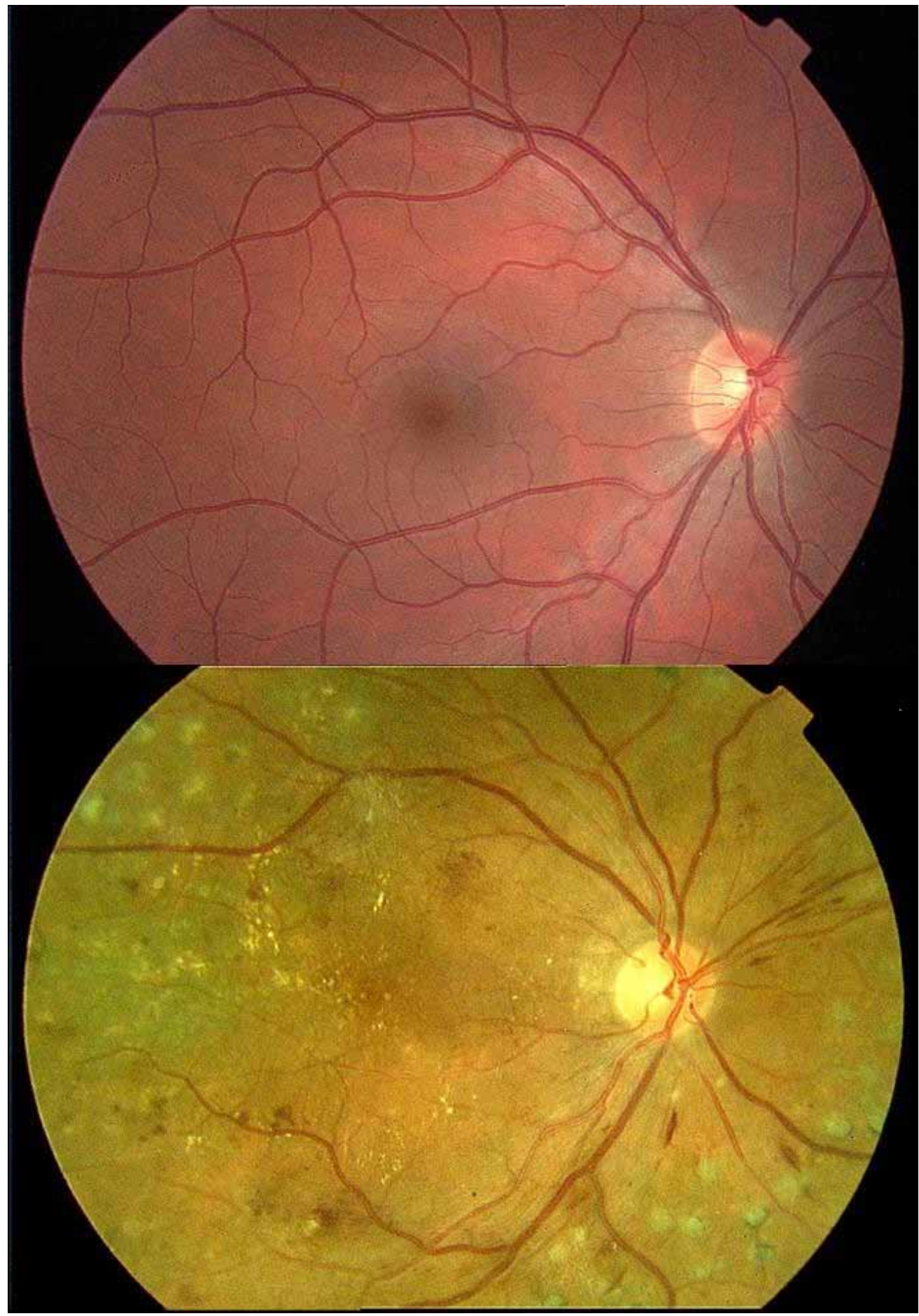


En resumen, la aplicación de programas de eOftalmologia basados en la retinografía no midriática para la detección de retinopatía diabética constituye un método muy extendido en la actualidad, cuya fiabilidad ha sido demostrada y que si se compara con los métodos clásicos de exploración retiniana, además de presentar algunas ventajas, exhibe una mejor relación coste-efectividad.

\section{Glaucoma crónico}

El glaucoma crónico es una neuropatía óptica progresiva asociada a una pérdida progresiva de campo visual y que con elevada frecuencia asocia una presión intraocular elevada ${ }^{14}$. Su prevalencia en la población oscila entre el 1 y el $3 \%$, es una causa importante de ceguera legal y un relevante problema de salud pública puesto que genera un elevadísimo número de consultas oftalmológicas. El diagnóstico y el seguimiento de esta enfermedad se realizan mediante la medida de la presión intraocular, el examen de la papila óptica y la determinación del estado del campo visual a través de los sistemas de campimetría ${ }^{15}$.

La detección y el seguimiento del glaucoma crónico es un proceso más complejo que el cribado de la retinopatía diabética puesto que precisa varios dispositivos diagnósticos. A pesar de ello, se han realizado intentos para establecer sistemas de eOftalmología para el manejo de esta enfermedad. Así, se ha valorado la posibilidad de diagnosticar el glaucoma mediante el examen de fotografías digitales de la papila obtenidas en consultas de atención primaria y remitidas a especialistas ${ }^{16}$. También se ha estudiado la utilización de DICOM para transmitir imágenes de papilas glaucomatosas y parece que su interpretación no se vería afectada por la captura y transmisión mediante este sistema ${ }^{17}$.

Los resultados obtenidos con la interpretación de imágenes digitales de la papilla son prometedores, pero el diagnóstico del glaucoma es un proceso mucho más complejo y no puede limitarse a esta prueba (Fig. 3). Por ello algunos autores se han planteado la posibilidad de establecer programas de eOftalmología para el cribado del glaucoma crónico mediante la toma de la presión intraocular, el examen de imágenes digitales de la papila y la realización del campo visual. Estas pruebas serían realizadas en localizaciones remotas o por personal no médico y posteriormente serían evaluadas por oftalmólogos que en función de los resultados determinarían qué pacientes pueden padecer glaucoma crónico y deben ser sometidos a un seguimiento más riguroso ${ }^{18,19}$.

Figura 3. Exploraciones en el glaucoma crónico. Izquierda: toma de la tensión intraocular. Centro: fotografía digital de la papila. La flecha blanca indica el borde de la papila, la flecha negra el borde de la excavación papilar. Derecha: campo visual de un paciente glaucomatoso. Las zonas negras corresponden a áreas de pérdida irreversible del campo visual.

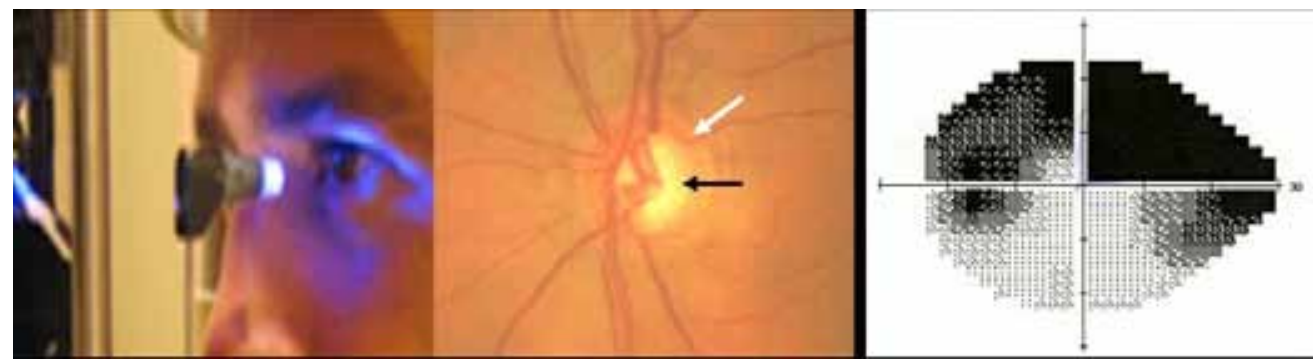

Los intentos por implementar programas de eOftalmología para el seguimiento de pacientes ya diagnosticados de glaucoma han sido muy escasos. Existe un estu- dio en el que se evaluó la posibilidad de realizar controles a distancia de la presión intraocular en pacientes glaucomatosos mediante dispositivos portátiles. Los datos 
serían enviados posteriormente al oftalmólogo responsable para que hiciese los ajustes necesarios ${ }^{20}$. El precio de los dispositivos portátiles sería un inconveniente de este método, pero no debemos olvidar que el seguimiento de esta enfermedad tampoco puede limitarse a la toma de la presión intraocular. En otro estudio piloto más ambicioso realizado en Finlandia se valoró el realizar el seguimiento de pacientes glaucomatosos residentes en zonas rurales combinando el examen mediante lámpara de hendidura conectada a un sistema de vídeo, la realización del campo visual y la toma de imágenes de la papila. La satisfacción de los pacientes fue muy alta y parece ser que este método supone un importante ahorro en tiempo y en costes derivados de los desplazamientos a los centros de atención especializada ${ }^{21}$.

Figura 4. Exploraciones en un paciente con DMAE. Arriba izquierda: fotografía digital de la retina. La flecha indica la zona de neovascularización subretiniana. Arriba derecha: angiografía fluoresceínica. Abajo: tomografía óptica de coherencia. La flecha indica la zona de acúmulo de líquido intrarretiniano.

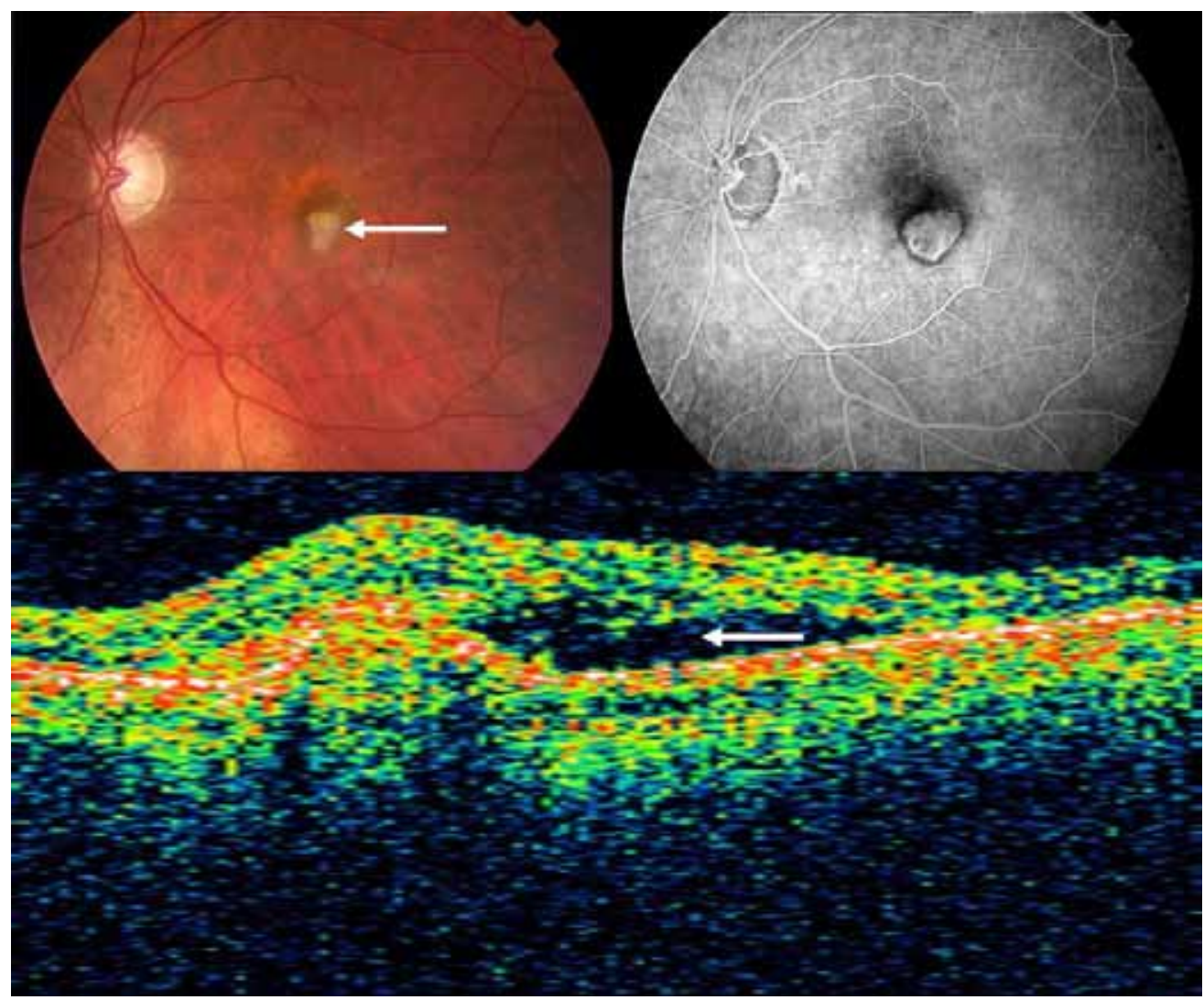

\section{Degeneración macular asociada a la edad}

La DMAE es un proceso degenerativo de la parte central de la retina, la mácula, que ocurre en personas de edad avanzada. Exis- ten dos formas, una atrófica o seca y otra exudativa o húmeda. La primera es más frecuente, menos invalidante y hasta la fecha no tiene tratamiento. La forma exudativa es menos frecuente y se caracteriza por la formación de neovasos subretinianos. Pue- 
de provocar una pérdida severa de la visión central y actualmente se trata mediante la inyección intravítrea de sustancias antiangiogénicas ${ }^{22}$. También la DMAE es un importante problema de salud pública puesto que si no se detecta y se trata de forma temprana va a provocar una disminución visual rápida, severa e irreversible. Es la primera causa de ceguera en pacientes de más de 65 años y es de suponer que, al ser una enfermedad relacionada con la edad, su prevalencia aumente en los próximos años con el envejecimiento de la población.

Igual que con las dos patologías anteriores, también con la DMAE se ha realizado algún intento por poner en marcha programas de eOftalmología para su diagnóstico. Así, algunos autores han estudiado la utilización de fotografías digitales de la retina para diagnosticar o clasificar esta enfermedad ${ }^{23-25}$ o para detectar lesiones susceptibles de ser $\operatorname{tratadas}^{26,27}$. Las fotografías digitales obtenidas en centros remotos y remitidas para que fuesen evaluadas por especialistas en retina podrían ser útiles para realizar el cribado de esta enfermedad y permitirían un acceso más rápido de los pacientes a los lugares de tratamiento ${ }^{25}$.

También estos resultados son alentadores pero, al igual que el glaucoma crónico, la DMAE es un proceso complejo cuyo diagnóstico y seguimiento requiere, además del examen de la retina, la utilización de otros dispositivos diagnósticos como son la angiografía fluoresceínica (AGF) o la tomografía óptica de coherencia (OCT) ${ }^{28}$ (Fig. 4).

\section{REQUERIMIENTOS TECNOLÓGICOS}

\section{Dispositivos diagnósticos}

Cada una de las patologías oculares que se han ido tratando requiere tanto para su diagnóstico como para su seguimiento diferentes dispositivos diagnósticos. Aunque para realizar el cribado de la retinopatía diabética puede ser suficiente la utilización de los retinógrafos, si lo que se quiere es poner en marcha programas de e-Oftalmología para el diagnóstico exacto y completo de esta patología se deberá incluir también la medición de la agudeza visual y el examen mediante OCT y AFG. En el glaucoma crónico se debe introducir la toma de la presión intraocular y la campimetría y en la DMAE es importante la medición de la agudeza visual y el examen mediante la OCT y la AGF. Los dispositivos diagnósticos pueden ir variando en el tiempo por la aparición de nuevos recursos o nuevas indicaciones diagnósticas en las patologías ya existentes. La característica común es que todos ellos deben producir imágenes digitales o susceptibles de ser digitalizadas. Además, es crucial que estas imágenes adopten el formato DICOM. Si los aparatos de diagnóstico no producen imágenes en DICOM, se deberá adaptar a los mismos conversores que transformen los datos a este formato.

\section{DICOM (Digital imaging and communication in medicine)}

El formato DICOM es el estándar reconocido mundialmente para el almacenamiento, transmisión e intercambio de imágenes médicas ${ }^{29}$. Aunque inicialmente se desarrolló para radiología, posteriormente se ha extendido a otras especialidades y desde 1999 incluye específicamente las características de los instrumentos oftalmológicos. DICOM define el formato de los archivos y los protocolos de comunicación dentro de la red. Esto facilita el intercambio de imágenes médicas entre equipos de diferentes fabricantes y permite la creación de bases de datos que se pueden comunicar con otros equipos que también se ajusten a su estándar.

Un archivo DICOM, además de los elementos multimedia que contiene (imagen, audio, vídeo), incluye otros atributos que describen el contexto de la captura para facilitar su manejo dentro de un sistema de eOftalmología. Estos atributos se agrupan en módulos, que son básicamente los mismos para todas las especialidades médicas:

- Información del paciente. Nombre, número de identificación, edad, sexo, etc. 
- Información sobre la exploración realizada. Lugar, fecha, usuario responsable, equipo empleado para la captura, etc.

- Información que identifica a cada archivo dentro de series que pueden incluir archivos similares.

- Información sobre los datos multimedia. Tipo de datos, formato, etc.

\section{Sistemas de almacenamiento, gestión y distribución de los datos del paciente y las imágenes médicas}

Son fundamentalmente dos:

- PACS (Picture archiving and communication system). Es el sistema de gestión, almacenamiento y visualización de imágenes médicas y de archivos DICOM. Puede incluir herramientas de procesado o de ayuda al diagnóstico. El PACS recibe la información de los dispositivos diagnósticos, la almacena y la presenta a los usuarios cuando estos la requieren.

- OIS (Ophthalmological information system). Es el sistema que almacena y distribuye toda la información de los pacientes en lo que se refiere a datos demográficos, estudios anteriores o citaciones.

Ambos deben estar interconectados y compartir e intercambiar la información y a su vez, también deben estar conectados a los sistemas electrónicos de historia clínica. El esquema de funcionamiento de todo el sistema se expone en las figuras 5 y 6 .

Figura 5. Esquema del funcionamiento de un sistema de eOftalmología en modo real o en modo diferido.

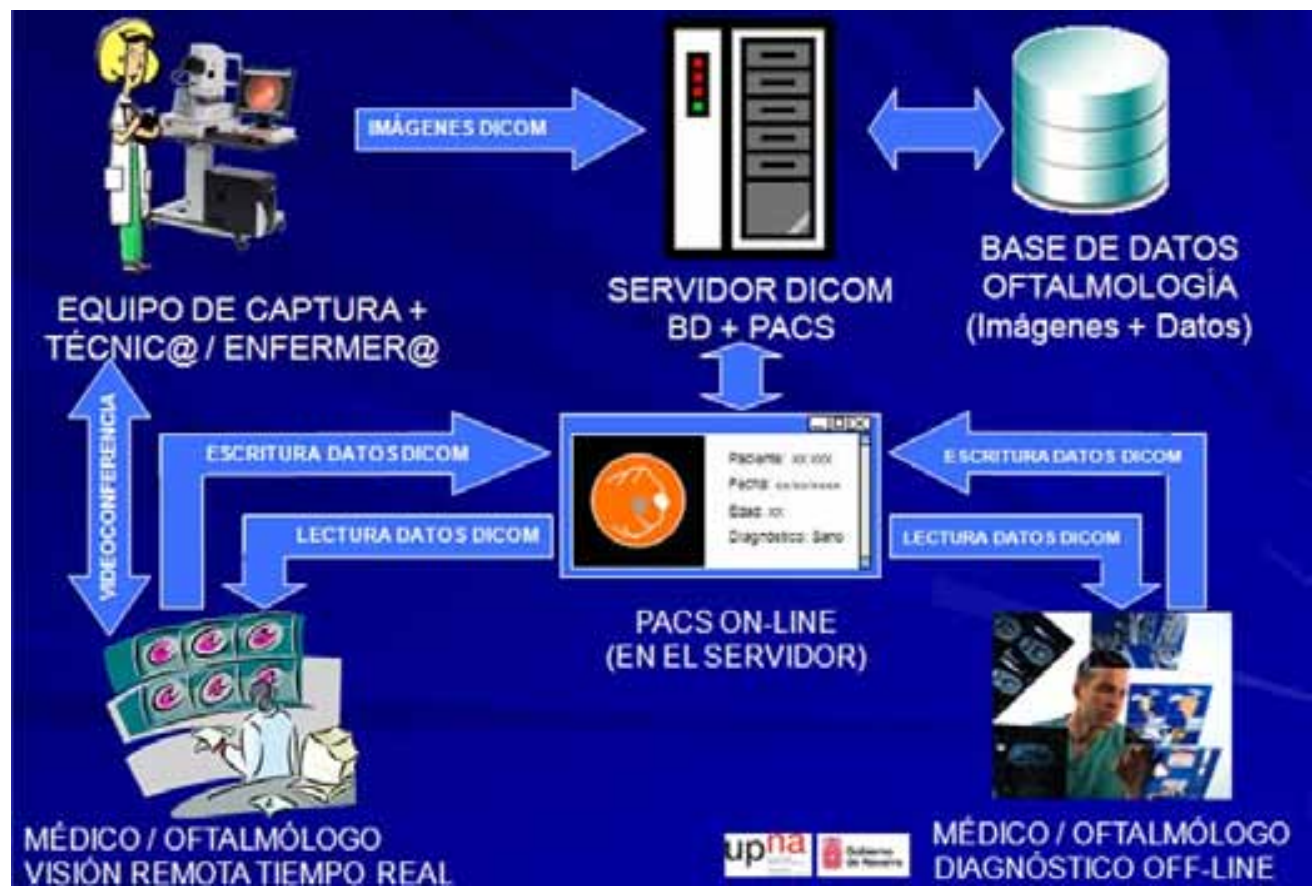


Figura 6. Esquema general del sistema de eOftalmología.

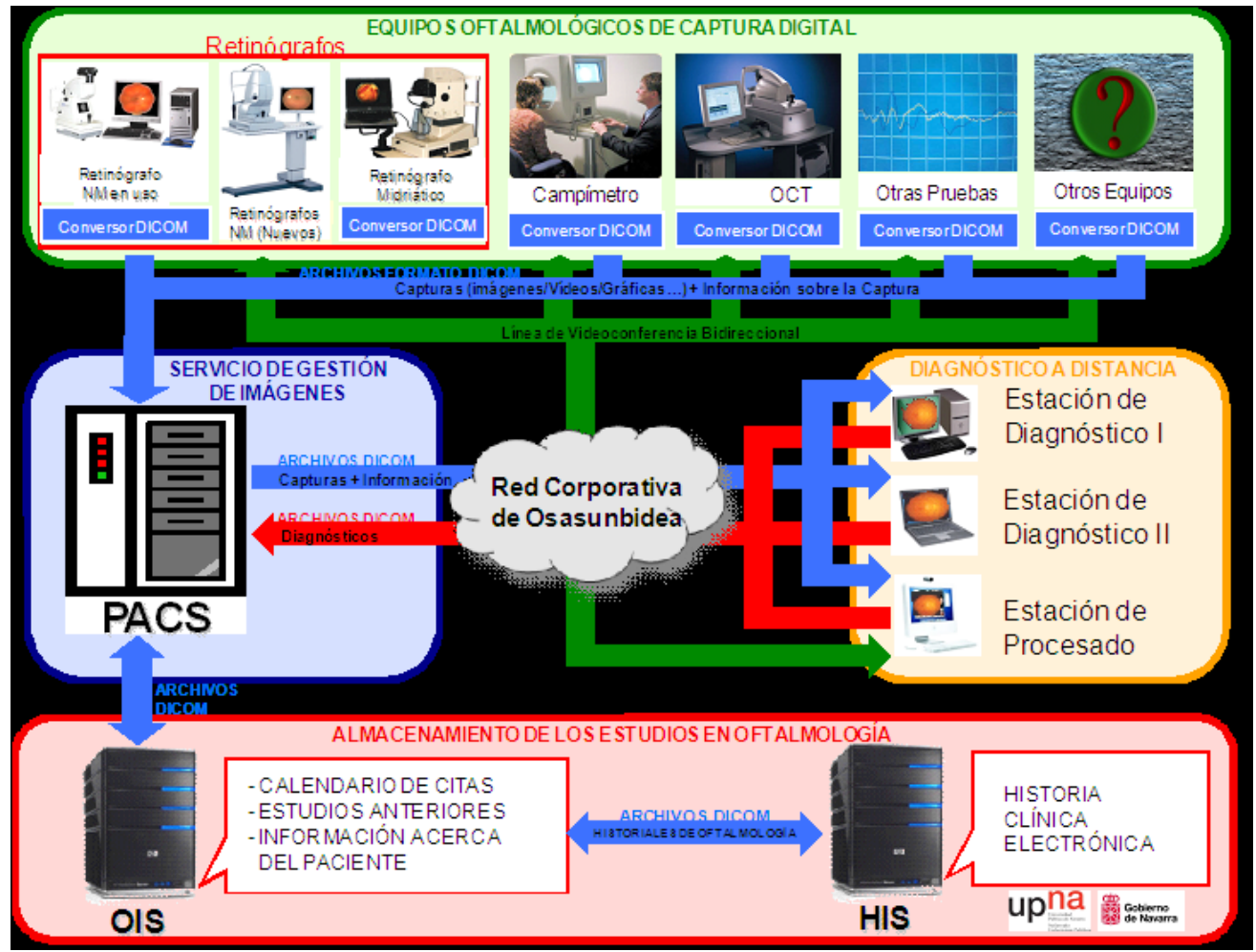

\section{VENTAJAS DE LOS SISTEMAS DE eOFTALMOLOGÍA}

Desde el punto de vista tecnológico, la digitalización de las imágenes y los datos médicos y su integración en una localización única tiene las siguientes ventajas:

- Mayor facilidad y comodidad para la consulta en cualquier momento y desde cualquier ordenador.

- Posibilidad de consulta o de trabajo a distancia (tele-oftalmología).

- Posibilidad de aplicar a las imágenes programas informáticos para su estudio, comparación o análisis evolutivo (ayudas al diagnóstico).

- Posibilidad de integrar diferentes exploraciones, imágenes y otros datos, en procesos diagnósticos o evolutivos.
- Facilidad y rapidez para la realización de estudios estadísticos de todo tipo de patologías relacionadas con la oftalmología.

Por otra parte, las ventajas desde el punto de vista asistencial son las siguientes:

- El diagnóstico o el seguimiento de algunas patologías oculares podría estar basado en el examen de imágenes obtenidas de los dispositivos tecnológicos de diagnóstico, junto con el resultado de exploraciones sencillas (agudeza visual, tensión ocular). Las pruebas serían realizadas por personal de enfermería o diplomados en óptica. Los médicos podrían llevar a cabo un análisis integral a distancia de las imágenes y/o de los resultados de las exploraciones. 
- Gracias a este modelo de trabajo no presencial, el especialista emplea menos tiempo con cada paciente. Puede ser útil en muchas patologías y puede disminuir las listas de espera y la presión en las consultas.

- La aplicación de programas informáticos en la organización de la asistencia permite optimizar aún más los recursos mediante la introducción de sistemas de alarma, de gestión de citas, agrupamiento de exploraciones por patologías, etc.

- Al trabajar con exploraciones digitalizadas pueden añadirse servicios como el de ayudas para el diagnóstico, basado en software de detección automática de patrones o en el tratamiento digital de la información.

\section{TENDENCIAS FUTURAS}

El aumento en la esperanza de vida de la población va a provocar un incremento en la demanda de atención sanitaria en los próximos años. La carencia de médicos que ya es patente en muchos países empeorará aún más esta situación, por lo que parece claro que se deben buscar soluciones que se apoyen en una utilización más intensiva de los recursos tecnológicos. Es posible que el modelo clásico de relación directa médico-paciente se vea sustituido por otro en el que entre el médico y el enfermo se interpongan los dispositivos diagnósticos y los recursos tecnológicos. Este proceso va a ser evidente en muchas especialidades, pero se verá especialmente acentuado en oftalmología, una especialidad basada en el análisis de las imágenes. Ya en la actualidad, sistemas como la OCT resultan imprescindibles para el seguimiento de pacientes con $\mathrm{DMAE}^{28} \mathrm{o}$ edema macular diabético ${ }^{30,31}$. En el glaucoma crónico, sistemas como la OCT, la polarimetría con láser de barrido (GDx) o la oftalmoscopía confocal con láser de barrido (HRT) parecen prometedores para monitorizar los cambios en la configuración de la papila óptica o en la capa de fibras nerviosas de la retina ${ }^{32-34}$. Parece claro que con los actuales recursos tecnológicos resulta factible establecer programas de eOftalmología que combinen varias exploraciones para el seguimiento de patologías como la retinopatía diabética, la DMAE o el glaucoma crónico ${ }^{35}$.

Los dispositivos tecnológicos de diagnóstico serán cada vez más precisos y más procesos podrán beneficiarse de esta situación. También los sistemas de transmisión y almacenamiento de imágenes mejorarán su eficacia. El incremento en la utilización de las nuevas tecnologías hará que su precio de adquisición se abarate y permitirá a más grupos incorporarse a ellas, generándose así una enorme cantidad de datos médicos que deberán ser almacenados y analizados. El manejo, la transmisión y el almacenamiento de estos datos va a tener un papel trascendental en la futura organización de la asistencia médica.

Este nuevo escenario va a provocar un remodelado en la asistencia sanitaria basado en una redistribución de tareas. Así, las enfermeras o los técnicos estarán a cargo de exploraciones médicas sencillas ${ }^{36} \mathrm{o}$ del manejo de los dispositivos diagnósticos. Los diferentes profesionales sanitarios trabajarán de forma independiente, en ocasiones en horarios y localizaciones diferentes, pero interconectados. Los oftalmólogos deberán reestructurar su trabajo y concentrarse en aquellas tareas en las que su presencia resulta indispensable. Serán otros profesionales sanitarios, bajo la supervisión a distancia de los oftalmólogos, quienes controlen a los pacientes en aquellas situaciones en las que la presencia de un especialista no sea esencial.

Este nuevo modelo de asistencia tiene ventajas tanto para los médicos como para los pacientes. Los especialistas optimizarán su tiempo de trabajo puesto que para ellos resulta más rápido valorar las imágenes en una interfaz gráfica que visitar a los pacientes en la consulta. La utilización de sistemas de eOftalmología en modo diferido les permitirá organizar su trabajo de una forma más personalizada e independiente del lugar y el tiempo. Además, la intercomunicación y el intercambio de in- 
formación entre especialistas de diferentes instituciones serán mucho más sencillo, con lo que cualquier nuevo avance será aplicado de forma más rápida, incluso en localizaciones remotas, promoviéndose así una especie de globalización científica. También será más sencilla la implantación de protocolos o de guías de práctica clínica o la realización de estudios multicéntricos con grandes grupos de pacientes ${ }^{37}$. La mayor utilización de archivos digitales hará que se desarrollen dispositivos informáticos de ayuda al diagnóstico. El aumento de la velocidad de procesado de los ordenadores hará que estos dispositivos sean cada vez más sensibles y específicos, optimizando así aún más el tiempo de trabajo de los especialistas.

Por lo que respecta a los pacientes, la implementación de modelos de eOftalmología supondrá un manejo más racional de sus problemas por parte de los sistemas sanitarios. Los pacientes podrán implicarse de una forma más activa en el seguimiento de su enfermedad y es de prever un mejor control de las listas de espera. Además, la implantación de programas masivos de cribado en diferentes patologías se realizará de forma más sencilla ${ }^{38}$. Puede ocurrir que la disminución del contacto directo entre los especialistas y los pacientes haga que éstos puedan pensar que su proceso no está siendo controlado de forma adecuada. Aquí será importante el papel de las enfermeras o del resto de los trabajadores sanitarios, asegurando a los enfermos que el médico responsable controlará el resultado de las exploraciones en un periodo de tiempo breve. El envío a los pacientes de informes escritos o de correos electrónicos informándoles de la evolución de su enfermedad hará que se sientan más seguros con este sistema.

No hay duda de que para alcanzar estos objetivos es fundamental el desarrollo de sistemas de eOftalmología rápidos y de manejo sencillo. El principal problema a la hora de poner en marcha estos modelos no está en la tecnología sino en un cambio de mentalidad por parte tanto de los pacientes como de los médicos.

\section{BIBLIOGRAFÍA}

1. Kumar S, Yogesan K. Internet-based eye care: VISION 2020. Lancet 2005; 366: 1244-1245.

2. Tang RA, Morales M, Ricur G, Schiffman JS. Telemedicine for eye care. J Telemed Telecare 2005; 11: 391-396.

3. American Diabetes Association. Standards of medical care for patients with diabetes mellitus. Diabetes Care 2000; 23: S32-S42.

4. Hernáez-Ortega MC, Soto-Pedre E, Vázquez JA, GutiérRez MA, Asua J. Estudio de la eficiencia de una cámara de retina no midriática para el diagnóstico de retinopatía diabética. Rev Clin Esp 1998; 198: 194-199.

5. Andonegui J, Berástegui L, SerranoL, Eguzkiza, A, Gaminde I, Aliseda D. Concordancia en el estudio de retinografías en diabéticos: oftalmólogos vs médicos de familia. Arch Soc Esp Oftalmol 2008; 83: 527-532.

6. Stellingwerf C, Hardus P, Hoooymans J. Twofield photography can identify patients with vision-threatening diabetic retinopathy. Diabetes Care 2001; 24: 2086-2090.

7. Lin YL, Blumenkranz MS, Brothers RJ, GrosVENOR DM. The sensitivity and specificity of single-field nonmydriatic monochromatic digital fundus photography with remote image interpretation for diabetic retinopathy screening: a comparison with ophthalmoscopy and standardized mydriatic color photography. Am J Ophthalmol 2002; 134: 204-213.

8. Ahmed J, Ward TP, Bursell SE, Aiello LM, CaVALLERANO JD, VIGERSKV RA. The sensitivity and specificity of nonmydriatic digital stereoscopic retinal imaging in detecting diabetic retinopathy. Diabetes Care 2006; 29: 2205-2209.

9. Gómez-Ulla F, Fernández MI, González F, Rey P, Rodríguez M, Rodríguez-Cid MJ et al. Digital retinal images and teleophthalmology for detecting and grading diabetic retinopathy. Diabetes Care 2002; 25: 1384-1389.

10. López-Bastida J, Cabrera-López F, Serrano-AguiLAR P. Sensitivity and specificity of digital retinal imaging for screening diabetic retinopathy. Diabet Med 2007; 24: 403-407.

11. Orruño Aguado E, Lapuente Troncoso JL, GutiérRez Iglesias A, Asua Batarrita J. Análisis de la introducción de la telemedicina en la gestión-coordinación de atención primariaespecializada. Evaluación de resultados y costes de experiencias preexistentes (teleoftalmología). Madrid: Plan Nacional para el SNS del MSC. Servicio de Evaluación de Tecnologías Sanitarias del País Vasco; 2007. 
Informes de Evaluación de Tecnologías Sanitarias: OSTEBA № 2006/07.

12. López Bastida J, Soto Pedre E, Cabrera López FA. Revisión sistemática y análisis coste efectividad del cribado de retinopatía diabética con retinografo no midriático mediante imágenes interpretadas por oftalmólogos frente a médicos de familia. Madrid: Plan Nacional para el SNS del MSC. Servicio de Evaluación del Servicio Canario de Salud; 2007. Informes de Evaluación de Tecnologías Sanitarias: SESCS № 2006/11.

13. Sender MJ, Montserrat S, Badia X, Maseras M, De la Puente ML, Foz M. Non mydriatic retinal camera: cost-effectiveness study for early detection of diabetic retinopathy. Med Clin (Barc) 2003; 121: 446-452.

14. Clasificación y terminología. En: European Glaucoma Society. Terminología y pautas para el glaucoma. Savona: Dogma 2009: 93-113.

15. American Academy of Ophthalmology. Primary open-angle glaucoma, preferred practice pattern. San Francisco: American Academy of Ophthalmology, 2005. Disponible en: www.aao.org/ppp

16. Li HK, TAng RA, Oschner K, Koploos C, Grady J, CRUMP WJ. Telemedicine screening of glaucoma. Telemedicine Journal 1999; 5: 283-290.

17. Khouri AS, Szirth BC, SAlti HI, Fechtner RD. DICOM transmission of simultaneous stereoscopic images of the optic nerve in patients with glaucoma. J Telemed Telecare 2007; 13 : 337-340.

18. Kumar S, Guibilato A, Morgan W, Jitskaia L, BaRRY C, BULSARA M et al. Glaucoma screening: analisis of conventional and telemedicinefriendly devices. Clinical Experimental Ophthalmology 2007; 35: 237-243.

19. Blázquez F, Sebastián MA, Antón A. Detección de glaucoma mediante telemedicina con SisGlaTel: satisfacción y aceptación entre los participantes y problemas detectados. Arch Soc Esp Oftalmol 2008; 83: 533-538.

20. Michelson G, Striebel W, Prihoda W, Schmidt W. Telemedicine in the control of intra-ocular pressure. J Telemed Telecare 2000; 6 (Supl 1): $126-128$.

21. Tullonen A, Ohinmaa T, Alanko Hi, Hyytinen P, JuUtinen A, Toppinen E. The application of teleophthalmology in examining patients with glaucoma: a pilot study. J Glaucoma 1999; 8: 367-373.

22. American Academy of Ophthalmology retina panel. Preferred practice pattern guidelines. Age-related macular degeneration. San Fran- cisco, CA: American Academy of Ophthalmology; 2008. Disponible en: http://www.aao. org/ppp

23. The age related eye disease study research group. The age related eye disease study system for classifying age-related macular degeneration from stereoscopic color fundus photographs: The age-related eye disease study report number 6 . Am J Ophthalmol 2001; 132: 668-681.

24. Scholl HPN, Dandekar SS, Peto T, Bunce C, Xing $\mathrm{W}$, JENKIns $\mathrm{S}$ et al. What is lost by digitizing stereoscopic fundus color slides for macular grading in age-related macular degeneration? Ophthalmology 2004; 111: 125-132.

25. Klein R, Meuer SM, Moss SE, Klein BEK, Neider MW, REINKE J. Detection of age-related macular degeneration using a nonmydriatic digital camera and standard film fundus camera. Arch Ophthalmol 2004; 122: 1642-1646.

26. Pirbhai A, Sheidow T, Hooper P. Prospective evaluation of digital non-stereo color fundus photography as a screening tool in age-related macular degeneration. Am J Ophthalmol 2005; 139: 455-461.

27. Le Tien V, Streho M, D’Athis P, Taillandier-HeriChe E, Paillaud E, Mahiddine $\mathrm{H}$ et al. Interobserver and intraobserver reliability of detecting age-related macular degeneration using a nonmydriatic digital camera. Am J Ophthalmol 2008; 146: 520-526.

28. Fung AE, Lalwani GA, Rosenfeld PJ, Dubovy SR, Michels S, Feuer WJ et al. An optical coherence tomography-guided, variable dosing regimen with intravitreal ranibizumab (Lucentis) for neovascular age-related macular degeneration. Am J Ophthalmol 2007; 143: 566-583.

29. Mildenberger P, Eichelberg M, Martin E. Introduction to the DICOM standard. Eur Radiol 2002; 12: 920-927.

30. KIм BY, SMITH SD, KAISER PK. Optical coherence tomographic patterns of diabetic macular edema. Am J Ophthalmol 2006; 142: 405-412.

31. Andonegui J, JimÉnEz-LASAntA L. Edema macular diabético. An Sist Sanit Navar 2008; 31 (Supl 3): 35-44.

32. Parikh RS, Parikh S, Sekhar GC, Kumar RS, PraBAKARAN S, BABU JG et al. Diagnostic capability of optical coherence tomography (StratusOCT 3) in early glaucoma. Ophthalmol 2007; 114: 2238-2243.

33. Reus NJ, De Graff M, Lemi HG. Accuracy of GDx VCC, HRT I and clinical assessment of stereoscopic optic nerve head photographs for diagnosing glaucoma. Br J Ophthalmol 2007; 91: 313-318. 
34. Medeiros FA, Vizzeri G, Zangwill LA, Alentar LM., Simple PA, WeinReb RN. Comparison of retinal nerve fiber layer and optic disc imaging for diagnosing glaucoma in patients suspected of having the disease. Ophthalmology 2008; 115: 1340-1346.

35. Andonegui J, Serrano L, Eguzkiza A. E-Health applications in ophthalmic diseases: on-going developments. En: Ma Manuela Cunha, Antonio Tavares y Ricardo Simöes. Handbook of research on developments in e-Health and telemedicine: technological and social perspectives. Hershey: Medical Information Science Reference (IGI Global), 2010: 1088-1115.
36. Soto MN, Silvestre MC, Andonegui J. Concordancia oftalmólogo-enfermera en la primera exploración postquirúrgica de la cirugía de cataratas. An Sist Sanit Navar 2008; 31: 241246.

37. Kennedy C, Kirwan J, Cook C, Roux P, Stulting A, Murdoch I. Telemedicine techniques can be used to facilitate the conduct of multicentre trials. J Telemed and Telecare 2000; 6: 343-347.

38. De Mul M, De Bont AA, Reus NJ, Lemij HG, Berg M. Improving the quality of eye care with tele-ophthalmology: shared-care glaucoma screening. J Telemed Telecare 2004; 10: 331336 . 
